\title{
Efficacy of Pulmonary Vein Isolation in Paroxysmal Atrial Fibrillation Patients With a Brugada Electrocardiogram
}

\author{
Takumi Yamada, MD; Yukihiko Yoshida, MD*; Naoya Tsuboi, MD**; \\ Yoshimasa Murakami, MD ${ }^{\dagger}$; Taro Okada, MD ${ }^{\dagger}$; Hugh T. McElderry, MD; \\ Naoki Yoshida, $\mathrm{MD}^{\dagger}$; Harish Doppalapudi, MD; Andrew E. Epstein, MD; \\ Vance J. Plumb, MD; Yasuya Inden, MD + ; \\ Toyoaki Murohara, MD ${ }^{\dagger \dagger}$ G. Neal Kay, MD
}

\begin{abstract}
Background Medical therapy of atrial fibrillation (AF) can be challenging in patients with Brugada electrocardiograms (ECGs). The purpose of this study was to investigate the efficacy of pulmonary vein (PV) isolation (PVI) in AF patients with Brugada ECGs.

Methods and Results PVI was performed in 6 consecutive patients exhibiting Brugada ECGs (type I in 1, type II in 4, and type III in 1) at baseline. In all patients exhibiting type II or III Brugada ECGs but 1, the administration of sodium-channel blockers converted those ECG patterns to a type I. Five of 6 (83\%) patients were free of symptomatic AF without any antiarrhythmic drugs after the first procedure. In the 1 remaining patient with $\mathrm{AF}$ recurrence and newly developed atrial tachycardia (AT), the residual conduction gaps of the 3 previously isolated PVs and a focal AT originating from the mitral isthmus were eliminated in the $2^{\text {nd }}$ session. Finally, during the follow-up period (11 \pm 6 months) after the last procedure, all patients were free of any symptomatic atrial arrhythmias without any antiarrhythmic drugs. No other complications occurred.

Conclusions Because of the concerns of proarrhythmias with antiarrhythmic drugs, PVI may be an effective strategy for highly symptomatic patients with AF who have a Brugada ECG pattern. (Circ J 2008; 72: 281-286)
\end{abstract}

Key Words: Atrial fibrillation; Brugada syndrome; Electrocardiogram; Pulmonary vein isolation; Radiofrequency catheter ablation

B rugada syndrome (BS) is characterized by an STsegment elevation in the right precordial leads of the electrocardiogram (ECG) and a high incidence of sudden cardiac death in patients with structurally normal hearts! It has been reported that approximately $20 \%$ of patients with BS develop supraventricular arrhythmias, and atrial fibrillation (AF) is associated in 10-20\% of those cases ${ }^{1,2}$ However, attempting medical control of the AF in those patients can be challenging because some antiarrhythmic drugs may increase the risk of sudden cardiac death! Idiopathic paroxysmal AF has been demonstrated to be triggered by atrial premature beats (APBs) originating from the pulmonary veins $(\mathrm{PVs})^{3}$ and catheter ablation to electrical-

(Received July 19, 2007; revised manuscript received August 28, 2007; accepted September 25, 2007)

Division of Cardiovascular Diseases, University of Alabama at Birmingham, Birmingham, AL, USA, *Division of Cardiology, Nagoya Daini Red Cross Hospital, Cardiovascular Center, **Division of Cardiology, Chukyo Hospital, Nagoya, 'Division of Cardiology, Aichi Prefectural Cardiovascular and Respiratory Center, Ichinomiya and tDepartment of Cardiology, Nagoya University Graduate School of Medicine, Nagoya, Japan

There was no financial support for this study.

Mailing address: Takumi Yamada, MD, Division of Cardiovascular Diseases, Cardiac Rhythm Management Laboratory, University of Alabama at Birmingham, VH B147, 1670 University Boulevard, 1530 3rd AVE S, Birmingham, AL 35294-0019, USA. E-mail: takumi-y @ fb4.so-net.ne.jp

All rights are reserved to the Japanese Circulation Society. For permissions, please e-mail: cj@j-circ.or.jp ly isolate the PVs from the left atrium (LA) has been established as an effective technique for controlling paroxysmal $\mathrm{AF}^{4-8}$ Because PV isolation (PVI) often eliminates the need for antiarrhythmic drugs to treat $\mathrm{AF}$, this procedure may be an especially attractive option for treating $\mathrm{AF}$ in patients with a Brugada ECG pattern. However, the efficacy of PVI is unknown in such patients, so the purpose of this study was to investigate the safety and efficacy of this technique.

\section{Methods}

\section{Patient Characteristics}

The subjects were 6 consecutive patients exhibiting a Brugada ECG at baseline who underwent an electrophysiologic study and catheter ablation for symptomatic AF. All patients had frequent attacks of paroxysmal (at least once a week) or persistent AF. The baseline characteristics of these patients, including the age, sex, echocardiographic parameters, presence of structural heart disease, Brugada ECG pattern, and nature of the clinical AF, were recorded. All patients gave written, informed consent before the procedure.

\section{Electrophysiologic Study}

The procedure was performed under sedation with intravenous propofol in the fasting state. For mapping and pacing, standard multielectrode catheters were placed in the coronary sinus and His bundle region. The transseptal procedure was performed with fluoroscopic or intracardiac echocardiography guidance. Catheterization into the LA 
was performed with a single-puncture, double-transseptal catheterization technique or 2 punctures, triple-transseptal catheterization technique ( 2 sheaths over 1 puncture site and the $3^{\text {rd }}$ sheath via a $2^{\text {nd }}$ puncture site) using an 8-Fr SL1 or 2 sheath (St Jude Medical, AF Division, Minnetonka, MN, USA). Intravenous heparin was administered to maintain an activated clotting time $>300 \mathrm{~s}$ after the atrial transseptal procedure. The ostial diameters of all $4 \mathrm{PVs}$ were measured by selective angiography in all cases as previously reported? The diameter of the PV ostium was measured across the PV antrum in each of the 2 projections (left and right anterior oblique views), and the mean value was obtained.

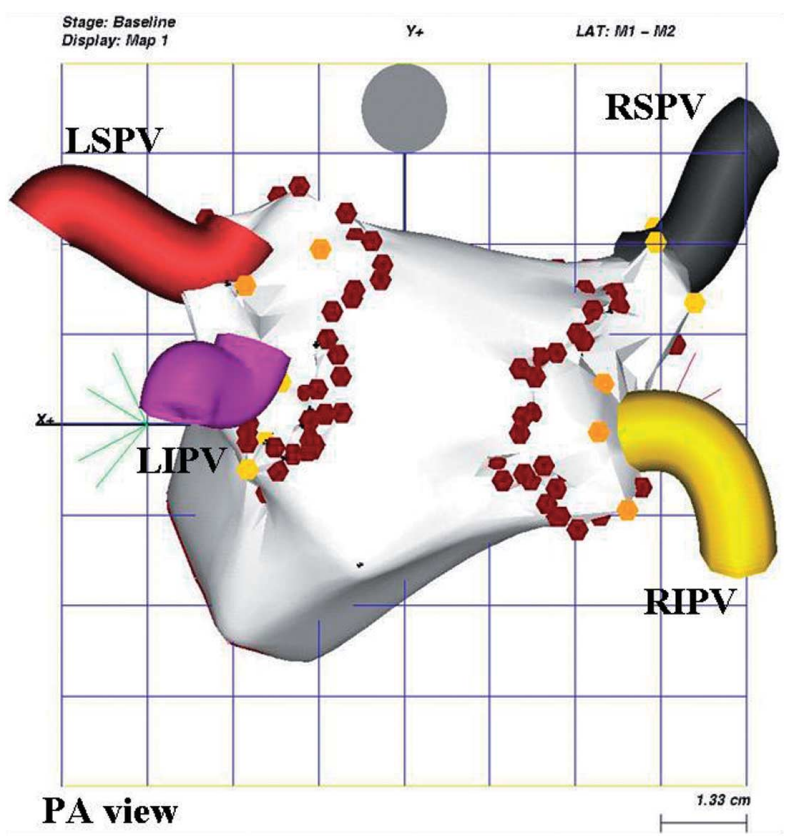

Fig 1. Electroanatomic map (postero-anterior (PA) view) showing the radiofrequency lesions created by encircling the ipsilateral pulmonary veins (PVs). The orange tag indicates the PV ostium and the red one the ablation site. LIPV, left inferior PV; LSPV, left superior PV; RIPV, right inferior PV; RSPV, right superior PV.

\section{PVI Technique}

In order to electrically isolate the PVs from the LA, either circumferential PV antrum isolation (CPVAI) or an encircling ipsilateral PVs isolation (EIPVsI) was performed using an electroanatomic mapping system $\left(\mathrm{CARTO}^{\mathrm{TM}}\right.$, Biosense-Webster, Diamond Bar, CA, USA) and multipolar circular mapping catheters. In the CPVAI, circumferential radiofrequency (RF) lesions were created approximately $1 \mathrm{~cm}$ away from the circular catheter positioned at the PV ostium. For the EIPVsI method, a 3-dimensional shell representing the LA was first constructed by an electroanatomic mapping system (Fig 1). RF current was then applied approximately $1 \mathrm{~cm}$ away from the double circular catheters positioned at the PV ostia to encircle the left- and right-sided ipsilateral PVs (Fig 1). RF energy was delivered to maintain an electrode temperature of less than $40^{\circ} \mathrm{C}$ with a maximum power setting of $40 \mathrm{~W}$ using a $3.5-\mathrm{mm}$ tip irrigated ablation catheter or with a target temperature of $55^{\circ} \mathrm{C}$ and maximum power setting of $40 \mathrm{~W}$ using a 4-mm tip nonirrigated ablation catheter. The end-point of these ablation techniques was complete PV electrical disconnection and non-inducibility of "spontaneous" AF during isoproterenol

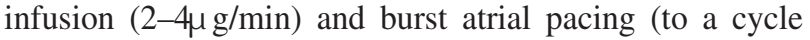
length as short as $200 \mathrm{~ms}$ ).

\section{Follow-up}

Follow-up was performed at 2 weeks, 1 month and every month thereafter, using 24-h Holter and cardiac recordings. All patients who reported symptoms were given an event monitor to document the cause of the symptoms. Computed tomography was performed before and 3-4 months after the ablation procedure to assess the PVs for stenosis.

\section{Results}

\section{Clinical Characteristics}

The baseline characteristics of the 6 patients are shown in Table 1 ( 5 males, 1 female; age range $42-61$ years). The echocardiographic dimension of the LA was $32-38 \mathrm{~mm}$ and left ventricular ejection fraction 55-75\%. None of the patients had any structural heart disease. The baseline ECG demonstrated a type I Brugada pattern in 1 patient, a type II pattern in 4 patients, and a type III pattern in 1 patient

Table 1 Clinical and Electrocardiographic Details of the Study Group

\begin{tabular}{|c|c|c|c|c|c|c|}
\hline & Patient 1 & Patient 2 & Patient 3 & Patient 4 & Patient 5 & Patient 6 \\
\hline Age & 54 & 59 & 42 & 61 & 43 & 59 \\
\hline Gender & $M$ & $M$ & $M$ & $F$ & $M$ & $M$ \\
\hline \multicolumn{7}{|l|}{$E C G$} \\
\hline LA diameter & $38 \mathrm{~mm}$ & $32 \mathrm{~mm}$ & $37 \mathrm{~mm}$ & $32 \mathrm{~mm}$ & $35 \mathrm{~mm}$ & $32 \mathrm{~mm}$ \\
\hline$L V E F$ & $55 \%$ & $66 \%$ & $60 \%$ & $70 \%$ & $75 \%$ & $64 \%$ \\
\hline$S H D$ & None & None & None & None & None & None \\
\hline \multicolumn{7}{|l|}{ Brugada syndrome } \\
\hline ECG type (spontaneous) & $I$ & II & II & II & II & III \\
\hline Na channel blocker test & $N / A$ & $(+)$ & $(+)$ & $(-)$ & $(+)$ & $(+)$ \\
\hline Family history & $(-)$ & $(-)$ & $(-)$ & $(+)$ & $(-)$ & $(-)$ \\
\hline Symptom & $(-)$ & Syncope (+) & $(-)$ & Syncope (+) & $(-)$ & Syncope (+) \\
\hline$V F$ induction & $(-)$ & $(+)$ & $(-)$ & $(-)$ & $N / A$ & $(+)$ \\
\hline ICD implantation & $(-)$ & $(+)$ & $(-)$ & $(+)$ & $(-)$ & $(+)$ \\
\hline \multicolumn{7}{|l|}{ Atrial fibrillation } \\
\hline $\begin{array}{l}\text { Type } \\
\text { Duration of symptoms }\end{array}$ & $\begin{array}{l}\text { Paroxysmal } \\
8 \text { years }\end{array}$ & $\begin{array}{l}\text { Paroxysmal } \\
3 \text { weeks }\end{array}$ & $\begin{array}{l}\text { Persistent } \\
14 \text { months }\end{array}$ & $\begin{array}{l}\text { Paroxysmal } \\
11 \text { months }\end{array}$ & $\begin{array}{l}\text { Paroxysmal } \\
18 \text { months }\end{array}$ & $\begin{array}{l}\text { Paroxysmal } \\
3 \text { months }\end{array}$ \\
\hline Atrial flutter & $(+)$ & $(+)$ & $(+)$ & $(+)$ & $(+)$ & $(+)$ \\
\hline
\end{tabular}

ECG, electrocardiogram; LA, left atrium; LVEF, left ventricular ejection fraction; SHD, structural heart disease; N/A, not available; VF, ventricular fibrillation; $I C D$, implantable cardioverter-defibrillator. 


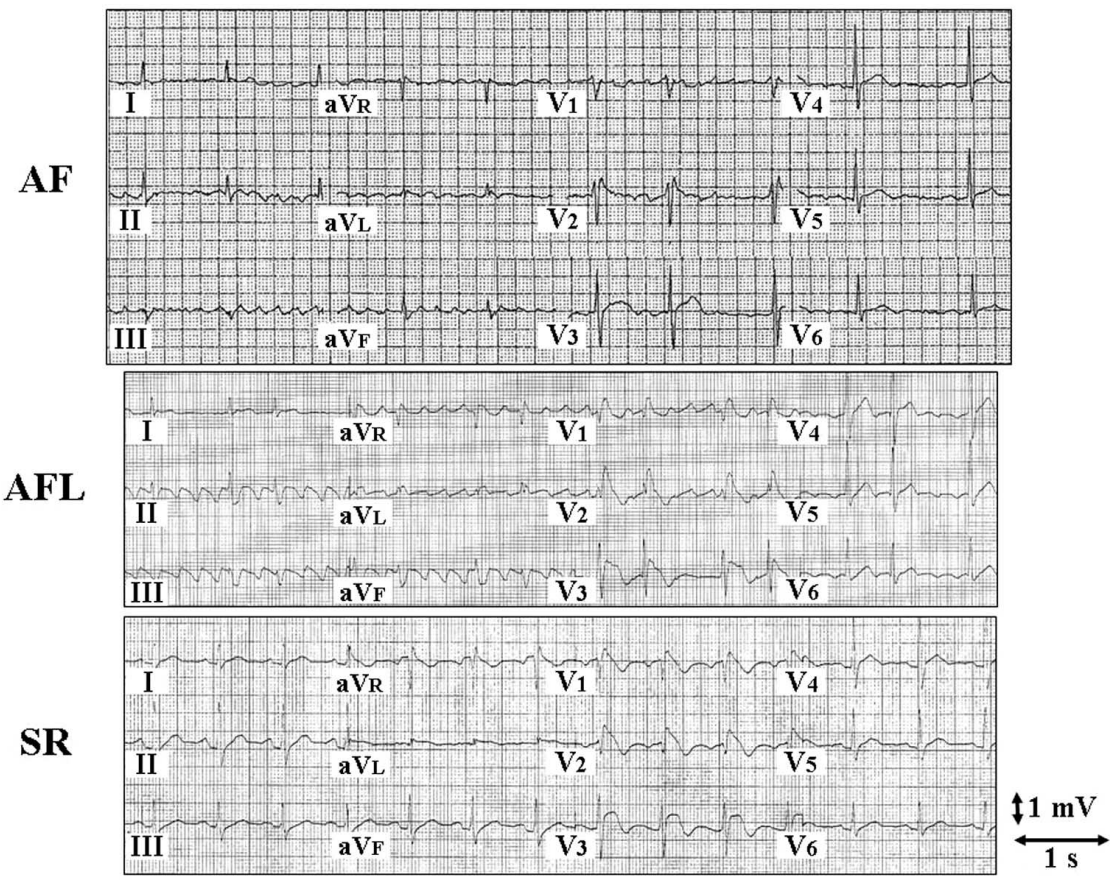

Fig 2. Type I Brugada electrocardiogram in Patient 1. AF, atrial fibrillation; AFL, atrial flutter; SR, sinus rhythm.
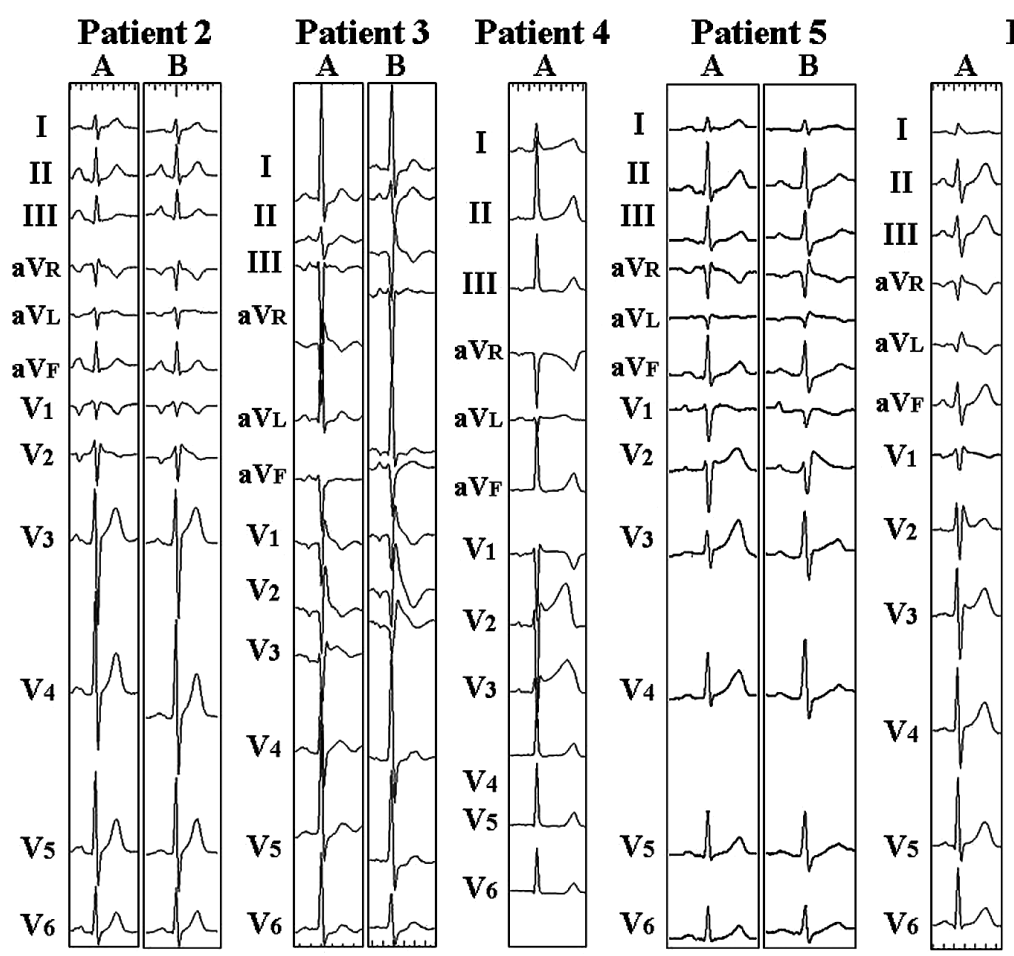

\section{Patient 6}

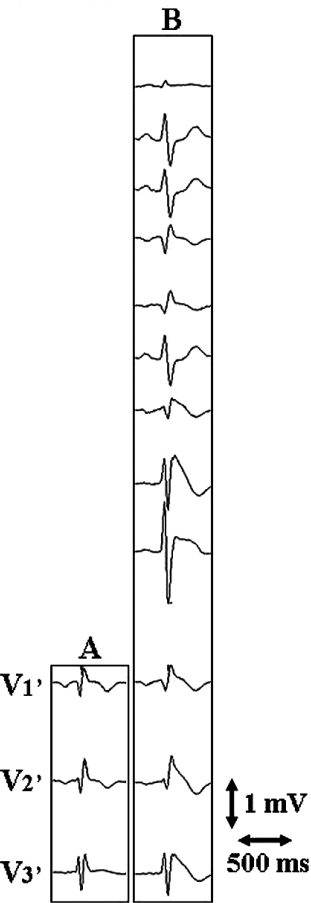

Fig 3. Brugada electrocardiograms in Patients 2-6. (A) Baseline, (B) after administration of a sodium-channel blocker. $\mathrm{V}_{1}, \mathrm{~V}_{2}$, and $\mathrm{V}_{3}$, , right precordial leads positioned at the $3^{\text {rd }}$ intercostal space.

(Figs 2,3). In all but 1 (Patient4) of the patients exhibiting a type II or III Brugada ECGs, the administration of a sodium-channel blocker (pilsicainide $1 \mathrm{mg} / \mathrm{kg}$ over $10 \mathrm{~min}$, IV ${ }^{10,11}$ ) converted the ECG to a type I pattern (Fig 3). Patient 4 alone had a family history of sudden cardiac death. Half of the patients had experienced syncope and in 2 of those patients, ventricular fibrillation was induced by programmed electrical stimulation. An implantable cardioverter-defibrillator (ICD) had been implanted in the 2 patients
(Patients 2 and 6), who satisfied the criteria for $\mathrm{BS}^{1}$ and in the 1 patient (Patient4) without a type I Brugada ECG, but with a history of syncope and family history of sudden death. All 3 patients had experienced inappropriate shocks from their ICD because of rapidly conducted AF. Five patients exhibited a paroxysmal form of $\mathrm{AF}$ and 1 had a persistent form. The AF duration was 3 weeks to 8 years. All the patients also had typical, cavotricuspid isthmusdependent atrial flutter. 
Table 2 Electrophysiological Details and Results of Catheter Ablation of the Study Group

\begin{tabular}{|c|c|c|c|c|c|c|}
\hline & Patient 1 & Patient 2 & Patient 3 & Patient 4 & Patient 5 & Patient 6 \\
\hline \multicolumn{7}{|l|}{$P V$ diameter $(\mathrm{mm})$} \\
\hline$L S P V$ & 21.6 & 19.3 & 18.9 & 22.4 & 18.5 & 17.4 \\
\hline$R S P V$ & 18.7 & 16.4 & 17.3 & 16.2 & 13.2 & 19.8 \\
\hline$L I P V$ & 16.9 & 18.0 & 13.3 & 14.6 & 13.8 & 14.1 \\
\hline$R I P V$ & 17.8 & 17.1 & 16.8 & 15.9 & 19.9 & 13.4 \\
\hline \multicolumn{7}{|l|}{ Ablation } \\
\hline Strategy & $C P V A+T A-I V C$ & EIPVs+TA-IVC & $E I P V s+T A-I V C$ & $C P V A+T A-I V C$ & EIPVs+TA-IVC & $E I P V s+T A-I V C$ \\
\hline$P V$ isolation & Success & Success & Success & Success & Success & Success \\
\hline$R F$ duration (min) & 42 & 48 & 54 & 72 & 63 & 58 \\
\hline Procedure time (min) & 157 & 145 & 128 & 155 & 151 & 138 \\
\hline Fluoroscopic time (min) & 53 & 50 & 42 & 58 & 46 & 45 \\
\hline \multicolumn{7}{|l|}{ Follow-up } \\
\hline Duration & 6 months & 18 months & 20 months & 11 months & 7 months & 5 months \\
\hline Recurrence & $(-)$ & $(-)$ & $(-)$ & $(-)$ & $(-)$ & $(+)$ \\
\hline Complications & $(-)$ & $(-)$ & $(-)$ & $(-)$ & $(-)$ & $L A A T$ \\
\hline
\end{tabular}

$P V$, pulmonary vein; $L S P V$, left superior PV; RSPV, right superior PV; LIPV, left inferior PV; RIPV, right inferior PV; CPVA, circumferential PV antrum ablation; TA-IVC, cavo-tricuspid isthmus ablation; EIPVs, encircling ipsilateral PVs; RF, radiofrequency; AT, atrial tachycardia. Other abbreviation see in Table 1.

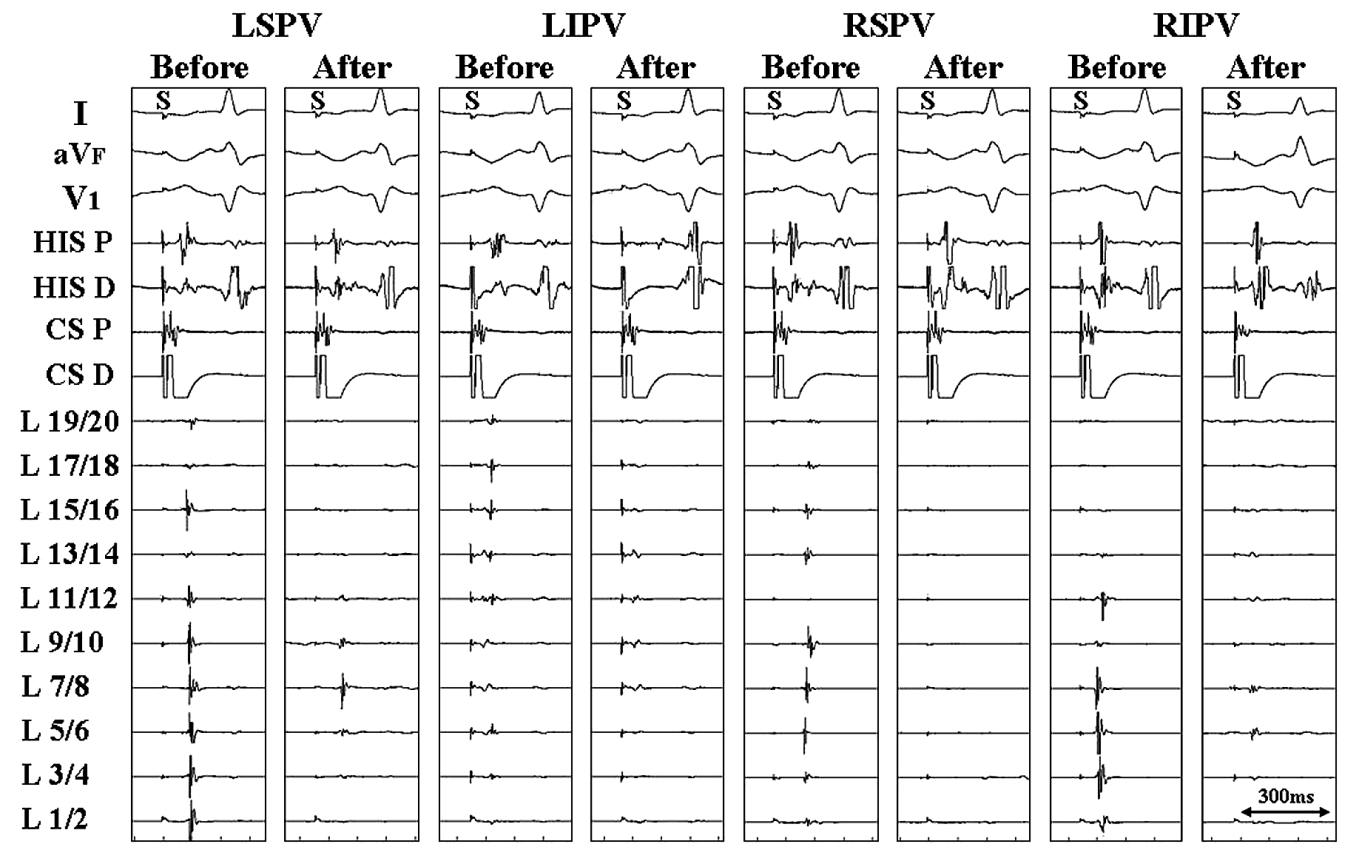

Fig 4. Successful PV electrical disconnection by circumferential PV antrum ablation during distal coronary sinus (CS) pacing. Note that the spiky potentials recorded from L 5/6 to 9/10 after the successful isolation of the LSPV reflect activation of the left atrial appendage. CS(HIS) D,P, the distal and proximal electrode pairs of the coronary sinus (His bundle) catheter; L1/2 to 19/20, the electrode pair of the circular catheter; S, spike from the distal CS pacing. Other abbreviations as in Fig 1.

\section{Electrophysiologic Study and Catheter Ablation}

The electrophysiologic findings and the results of catheter ablation are summarized in Table 2. The mean ostial diameter was $19.7 \pm 1.9$ (17.4-22.4), 16.9 2.3 (13.2-19.8), 15.1 \pm 1.9 (13.3-18.0), and 16.8 $\pm 2.1(13.4-19.9) \mathrm{mm}$ for the left superior PVs, right superior PVs, left inferior PVs, and right inferior PVs, respectively. At baseline, the heart rhythm was sinus rhythm in 2 patients, AF in 3 patients, and typical atrial flutter in 1 patient (Patient 1, Fig 2). In the 2 patients with sinus rhythm, AF was induced by burst atrial pacing from the coronary sinus and no APBs were observed after the spontaneous termination of AF. In the patient with atrial flutter, a cavotricuspid isthmus ablation was first performed and no APBs were observed after the termination of atrial flutter. In 2 of the 3 patients with AF, it was terminated spontaneously before ablation and thereafter no APBs were observed throughout the procedure. In the remaining patient with $\mathrm{AF}, \mathrm{PV}$ ablation was performed during AF. CPVAI was performed in 2 patients and EIPVsI in the remaining patient. In the 1 patient undergoing EIPVsI during AF, the AF terminated during the ablation of the left PVs. Successful PV electrical disconnection was achieved for all the PVs in all the patients (Figs 4,5). Neither vagal responses nor ST-segment elevation during PVI were observed in any of the patients. Thereafter, burst atrial pacing from the coronary sinus with an isoproterenol infusion induced AF in 1 patient and typical atrial flutter in another patient. In the patient with the induced AF, 

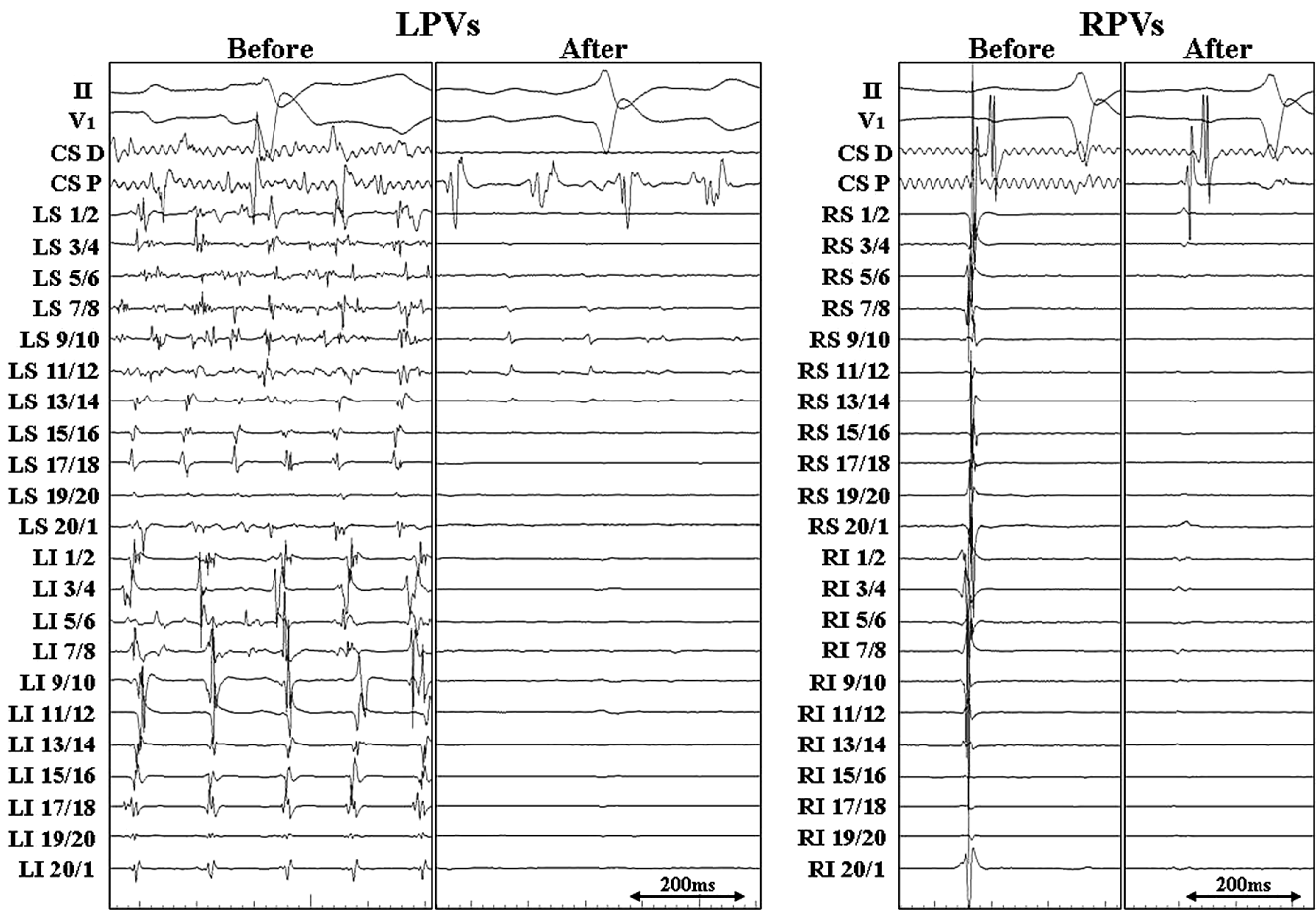

Fig 5. Successful PV electrical disconnection by encircling the ipsilateral PVs. LI, left inferior PV; LS, left superior PV; LPVs, left PVs; RI, right inferior PV; RPVs, right PVs; RS, right superior PV. Other abbreviations as in Fig 4.

cardioversion was performed and neither spontaneous AF nor APBs were observed after restoration of sinus rhythm. In the patient with the induced typical atrial flutter, a cavotricuspid isthmus ablation was performed and thereafter neither spontaneous AF nor APBs were observed. Cavotricuspid isthmus ablation was also performed in all of the other patients. The mean duration of RF application to complete the PV electrical disconnection was 56 \pm 11 (4272) $\min$. The total procedure and fluoroscopy times were $146 \pm 11(128-157)$ and 49 $\pm 6(42-58)$ min, respectively.

\section{Follow-up}

During follow-up, 5 of the $6(83 \%)$ patients were free of symptomatic AF without any antiarrhythmic drugs after the first procedure. The remaining patient (Patient6) had an early recurrence of AF and a newly developed atrial tachycardia (AT) after the first procedure and a $2^{\text {nd }}$ procedure was performed immediately. Recovery of an electrical connection was observed in the $3 \mathrm{PVs}$ and a few RF applications delivered at the conduction gaps with electrophysiologic guidance achieved successful electrical disconnection in all the PVs. However, even after that, clinical AT was still induced by burst atrial pacing from the coronary sinus. Detailed mapping of the LA revealed that the tachycardia was a focal AT originating from the mitral isthmus. After the AT was eliminated by a few RF applications, neither AF nor AT was induced despite burst atrial pacing with isoproterenol infusion. Finally, during the follow-up period (11 6 , 5-20 months) after the last procedure, 6 of 6 (100\%) patients were free of any symptomatic atrial arrhythmias without any antiarrhythmic drugs. No PV stenosis was observed and no other complications occurred.

\section{Discussion}

It is well known that $\mathrm{AF}$ is often observed in patients with $\mathrm{BS}^{1,2}$ and it can be a difficult clinical problem for those patients because attempts to control the AF with antiarrhythmic medications may increase the risk of sudden cardiac death. In addition, the use of an ICD may be complicated by inappropriate shocks because of the rapidly conducted AF in these patients. Because of these concerns, non-pharmacological therapy to eliminate the AF is especially attractive for patients with BS. Though a type I ECG, which is characterized by a coved-typed ST-segment elevation of $\geq 2 \mathrm{~mm}(0.2 \mathrm{mV})$ followed by a negative $\mathrm{T}$ wave, is necessary for a definitive diagnosis of BS, the ECG pattern can be dynamic and is often concealed! Therefore, AF patients with type II or III Brugada ECGs, which are characterized by a ST-segment elevation with a saddleback appearance and high takeoff of the ST-segment elevation $\geq 2 \mathrm{~mm}$, trough displaying an ST elevation $\geq 1 \mathrm{~mm}$, and then either a positive or biphasic $\mathrm{T}$ wave (type II) or either a saddleback or coved appearance with an ST-segment elevation $<1 \mathrm{~mm}$ (type III), may also be at risk for adverse effects of antiarrhythmic drugs. To the best of our knowledge, this is the first report to investigate the safety and efficacy of PVI in AF patients with a Brugada ECG pattern. We demonstrated that PVI was highly effective and safe in AF patients with a Brugada ECG, similar to other patients with idiopathic AF4-8,12 These findings suggest that PVI is a potential first-line therapy for symptomatic AF in patients with a Brugada ECG.

Morita et al reported that atrial vulnerability is enhanced in patients with BS and that abnormal atrial conduction may be an electrophysiologic basis for the induction of AF in patients with $\mathrm{BS}^{2}$. However, the mechanism of AF occurrence in the patients with $\mathrm{BS}$ or with a Brugada ECG alone remains unclear. In the present study there was no evidence that $\mathrm{AF}$ originated from the PVs in any of the patients; however, PVI alone eliminated AF completely in all of them. It has been reported that arrhythmogenic PVs for AF 
exhibit a dilatation of the ostium ${ }^{9}$ and in all of the present patients, the PV ostial diameters were larger than those of the arrhythmogenic PVs in the previous report? These findings also suggest that the PVs in all the patients in this study might have been arrhythmogenic for AF.

We cannot discuss the efficacy and safety of the PVI in each of the stratifications because the clinical characteristics regarding BS were variable in the present patients. Further studies are needed to clarify this issue, but we believe that the results of this study have significant clinical implications.

\section{Conclusion}

In this small group of patients with a Brugada ECG pattern, PVI appeared to be safe and effective, suggesting that this therapy deserves consideration for highly symptomatic individuals with paroxysmal and persistent AF.

\section{References}

1. Antzelevitch C, Brugada P, Borggrefe M, Brugada J, Brugada R, Corrado D, et al. Brugada syndrome: Report of the second consensus conference: Endorsed by the Heart Rhythm Society and the European Heart Rhythm Association. Circulation 2005; 111: 659-670.

2. Morita H, Kusano-Fukushima K, Nagase S, Fujimoto Y, Hisamatsu $\mathrm{K}$, Fujio $\mathrm{H}$, et al. Atrial fibrillation and atrial vulnerability in patients with Brugada syndrome. J Am Coll Cardiol 2002; 40: 1437-1444.

3. Haissaguerre M, Jais P, Shah DC, Takahashi A, Hocini M, Quiniou $\mathrm{G}$, et al. Spontaneous initiation of atrial fibrillation by ectopic beats originating in the pulmonary veins. N Engl J Med 1998; 339: 659666.
4. Haissaguerre M, Shah DC, Jais P, Hocini M, Yamane T, Deisenhofer I, et al. Electrophysiological breakthroughs from the left atrium to the pulmonary veins. Circulation 2000; 102: 2463-2465.

5. Ouyang F, Bansch D, Ernst S, Schaumann A, Hachiya H, Chen M, et al. Complete isolation of left atrium surrounding the pulmonary veins: New insights from the double-Lasso technique in paroxysmal atrial fibrillation. Circulation 2004; 110: 2090-2096.

6. Yamada T, Murakami Y, Okada T, Okamoto M, Shimizu T, Toyama $\mathrm{J}$, et al. Electrophysiological pulmonary vein antrum isolation with a multielectrode basket catheter is feasible and effective for curing paroxysmal atrial fibrillation: Efficacy of minimally extensive pulmonary vein isolation. Heart Rhythm 2006; 3: 377-384.

7. Yamane T, Date T, Kanzaki Y, Inada K, Matsuo S, Shibayama K, et al. Segmental pulmonary vein antrum isolation using the "large-size" lasso catheter in patients with atrial fibrillation. Circ $J$ 2007; 71: $753-760$.

8. Kumagai K, Noguchi H, Ogawa M, Nakashima H, Zhang B, Miura $\mathrm{S}$, et al. New approach to pulmonary vein isolation for atrial fibrillation using a multielectrode basket catheter. Circ J 2006; 70: 88-93.

9. Lin WS, Prakash VS, Tai CT, Hsieh MH, Tsai CF, Yu WC, et al. Pulmonary vein morphology in patients with paroxysmal atrial fibrillation initiated by ectopic beats originating from the pulmonary veins: Implications for catheter ablation. Circulation 2000; 101: 1274-1281.

10. Takenaka S, Emori T, Koyama S, Morita H, Fukushima K, Ohe T. Asymptomatic form of Brugada syndrome. Pacing Clin Electrophysiol 1999; 22: $1261-1263$

11. Shimizu W, Aiba T, Kurita T, Kamakura S. Paradoxic abbreviation of repolarization in epicardium of the right ventricular outflow tract during augmentation of Brugada-type ST segment elevation. $J$ Cardiovasc Electrophysiol 2001; 12: 1418-1421.

12. Ouyang F, Antz M, Ernst S, Hachiya H, Mavrakis H, Deger FT, et al. Recovered pulmonary vein conduction as a dominant factor for recurrent atrial tachyarrhythmias after complete circular isolation of the pulmonary veins: Lessons from double Lasso technique. Circulation 2005; 111: $127-135$. 